\title{
WAVELET DECOMPOSITION OF THE FINANCIAL MARKET
}

\author{
Lukáš Vácha, Miloslav Vošvrda*
}

\begin{abstract}
:
A heterogeneous agents model with the Worst Out Algorithm (WOA) was considered for obtaining more realistic market conditions. The WOA replaces periodically the trading strategies that have the lowest performance level of all strategies presented on the market by the new ones. New strategies that enter the market have the same stochastic structure as an initial set of strategies. This paper shows, by wavelets applications, measurement of influences of the trading strategies with the WOA.
\end{abstract}

Keywords: agents' trading strategies, heterogeneous agents model with stochastic memory, worst out algorithm, wavelet

JEL Classification: C61, G12, G14, D81

\section{Introduction}

Modern finance undergoes an important change of an economic agent perceiving, i.e., from a representative, rational agent approach towards a behavioural, agent-based approach in which markets represented by bindingly rational, heterogeneous agents using rule of thumb strategies. In the traditional approach, simple analytically tractable models with a representative, perfectly rational agent have been the main cornerstones and mathematics has been the main tool of analysis. The new behavioural approach fits much better with agent-based simulation models and computational and numerical methods have become an important tool of analysis (Hommes, 2006). The new behavioural, heterogeneous agents approach challenges the traditional representative, rational agent framework. Heterogeneity in expectations can lead to market instability and complicated dynamics of prices. Prices are driven by endogenous market forces. In the Heterogeneous Agents Model (HAM) typically two types of agents are distinguished, fundamentalists and chartists. Fundamentalists base their expectations about future asset prices and their trading strategies upon market fundamentals and economic factors, such as dividends, earnings, macroeconomic growth, unemployment rates, etc. Chartists or technical analysts try to extrapolate observed price patterns, such as trends, and exploit these patterns in their investment decisions. One of the expressive models was de-

* Institute of Information Theory and Automation, Academy of Sciences of the Czech Republic and Institute of Economic Studies, Faculty of Social Sciences, Charles University, Prague (vosvrda@utia.cas.cz)

A support from the Grant Agency of Charles University under the grant No. 454/2004/A EK/FSV, the Czech Science Foundation under the grant Nos. 402/04/1026 and 402/06/1417 is gratefully acknowledged. 
veloped by Brock and Hommes (Brocks, Hommes, 1998). In our early work (Vošvrda, 2001) we focused on a simple HAM with two or four types of belief that were fixed for all our simulations. In our previous papers (see Vošvrda, Vácha, $2002 \mathrm{a}$; and Vošvrda, Vácha, 2002 b, 2003), we employed a memory and some learning schemes in the Brock and Hommes's model. In this paper we use the core of the Brock and Hommes's model on which we base further extensions, such as a stochastic formation of beliefs and parameters including a memory length. Another extension is in an application of the Worst Out Algorithm (WOA). We show (see Vácha, Vošvrda, 2005), how a memory length distribution in the agents' performance measure affects a persistence of the generated price time series. Our motivation is to trace a memory length in the price time series with different replacement ratios of the improved WOA. The implementation of the WOA should increase a persistence of returns. A wavelet analysis is a convenient tool for an activity detection on various scales of the price time series. This one is more convenient tool for the frequency detection in the price time series than the Fourier analysis because the price changes in the financial market mood are better detected in time. The wavelet analysis uses time-scale domain instead of time-frequency domain. Financial markets have a typical stylized fact in existence clusters of both high positive returns and low negative returns in the realizations of the price time series. We can retrospectively analyse which part of the trading strategies set was used on the financial market and we can estimate their statistical properties.

\section{Model}

Financial markets are considered as systems of the interacting agents processing new information immediately. Prices are driven by endogenous market forces. Agents adapt their predictions by choosing among a finite number of predictors (see Abramovich, Bailey, Sapatinas, 1999). Each predictor has a performance measure. Based on this performance measure, agents realize a rational choice among the predictors (see Brock, Hommes, 1998). This approach relied on heterogeneity in the agent information and subsequent decisions either as fundamentalists or as chartists (see Chiarella, 1992; Chiarella, He, 2000). Let us consider an asset-pricing model with one risky asset and one risk-free asset. Let $p_{t}$ be the share price (ex dividend) of the risky asset at time $t$, and let $\left\{y_{t}\right\}$ be an i.i.d. stochastic dividend process of the risky asset. The risk free asset is perfectly elastically supplied and pays a fixed rate of return $r$. The gross risk-free interest rate $r^{g}$ is equal $1+r$. The risky asset pays a random dividend. The dynamics of wealth can then be written as

$$
\mathbf{W}_{t+1}=r^{g} \cdot W_{t}+\left(\mathbf{p}_{t+1}+\mathbf{y}_{t+1}-r^{g} \cdot p_{t}\right) \cdot z_{t},
$$

where $z_{t}$ denotes the number of shares of the asset purchased at time $t$, and a bold face type denotes random variable at date $t$. Let $E_{t}$ and $V_{t}$ denote the conditional expectation and conditional variance operators, based on the publicly available information set consisting of past prices and dividends, i.e., on the information set $F_{t}=\left\{p_{t}\right.$, $\left.p_{t-1}, \ldots ; y_{t}, y_{t-1}, \ldots\right\}$. Let $E_{h, t}, V_{h, t}$ denote forecasts of investor of type $h$ about the conditional expectation and conditional variance. Investors are supposed to be a myopic 
mean-variance maximizer so that the demand $\mathrm{z}_{h, t}$ for risky asset is obtained by solving the following criterion

$$
\max _{z_{i}}\left\{E_{h, t}\left[\mathbf{W}_{t+1}\right]-(a / 2) \cdot V_{h, t}\left[\mathbf{W}_{t+1}\right]\right\}
$$

where a risk aversion, $a$, is here assumed to be the same for all traders. Thus the demand $\mathrm{z}_{h, t}$ of type $h$ for risky asset has the following form

$$
\begin{gathered}
E_{h, t}\left[\mathbf{p}_{t+1}+\mathbf{y}_{t+1}-r^{g} \cdot p_{t}\right]-a \cdot \sigma^{2} \cdot z_{h, t}=0, \\
z_{h, t}=E_{h, t}\left[\mathbf{p}_{t+1}+\mathbf{y}_{t+1}-r^{g} \cdot p_{t}\right] /\left(a \cdot \sigma^{2}\right)
\end{gathered}
$$

assuming that the conditional variance of excess returns is a constant for all investor types

$$
V_{h, t}\left(\mathbf{p}_{t+1}+\mathbf{y}_{t+1}-r^{g} \cdot p_{t}\right) \equiv \sigma_{h}^{2}=\sigma^{2} .
$$

Let $\mathrm{z}^{\mathrm{s}}$ be a supply of outside risky shares per investor. Let $n_{h, t}$ be a fraction of type $h$ at date $t$. The equilibrium of demand and supply is

$$
\sum_{h=1}^{H} n_{h, t}\left\{E_{h, t}\left[\mathbf{p}_{t+1}+\mathbf{y}_{t+1}-r^{g} \cdot p_{t}\right] / a \cdot \sigma^{2}\right\}=z^{s}
$$

where $H$ is the number of different investor types. For the special case of zero supply, i.e., $z^{s}=0$, the market equilibrium is as follows

$$
r^{g} \cdot p_{t}=\sum_{h=1}^{H} n_{h, t} \cdot E_{h, t}\left[\mathbf{p}_{t+1}+\mathbf{y}_{t+1}\right]
$$

If there is only one investor type, i.e. $H=1$, the market equilibrium yields the following pricing equation

$$
r^{g} \cdot p_{t}=E_{t}\left[\mathbf{p}_{t+1}+\mathbf{y}_{t+1}\right] .
$$

It is well known that, using the arbitrage (1.7) repeatedly and assuming that the transversality condition

$$
\lim _{k \rightarrow \infty} \frac{E_{t}\left[\mathbf{p}_{t+1}\right]}{\left(r^{g}\right)^{k}}=0
$$

holds, a fundamental price of the risky asset is uniquely obtained by

$$
p_{t}^{*}=\sum_{k=1}^{\infty} \frac{E_{t}\left[\mathbf{y}_{t+k}\right]}{\left(r^{g}\right)^{k}} \text {. }
$$

Thus the fundamental price $p_{t}^{*}$ depends on the stochastic dividend process $\left\{y_{t}\right\}$. From the equation (1.7) we obtain the following price equation

$$
p_{i}=p_{t}^{*}+\left(r^{g}\right)^{t} \cdot\left(p_{0}-p_{0}^{*}\right) \text {. }
$$

For our purpose, it is better to work with the deviation $x_{t}$ from the benchmark fundamental price $p_{t}{ }^{*}$, i.e., $x_{t}=p_{t}-p_{t}{ }^{*}$. 


\section{Evolutionary Dynamics of Investors}

Let us admit the following assumptions:

A1)

$$
E_{h, t}\left[\mathbf{y}_{t+1}\right]=E_{t}\left[\mathbf{y}_{t+1}\right]
$$

A2)

$$
V_{h, t}\left(\mathbf{p}_{t+1}+\mathbf{y}_{t+1}-r^{g} \cdot p_{t}\right)=V_{t}\left(\mathbf{p}_{t+1}+\mathbf{y}_{t+1}-r^{g} \cdot p_{t}\right)=\sigma_{t}^{2},
$$

A3) all forecasts $E_{h, t}\left[\mathbf{p}_{t+1}\right]$ have the following form

$$
E_{h t}\left[\mathbf{p}_{t+1}\right]=E_{t}\left[\mathbf{p}_{t+1}^{*}\right]+f_{h}^{L}\left(x_{t-1}, \ldots, x_{t-L}\right) .
$$

Each forecast $f_{h}^{L}$ represents a model of the market for which type $h$ believes that prices deviate from the fundamental price. Let us concentrate on the evolutionary dynamics of the fractions $n_{h, t}$ of different $h$-investor types, i.e.

$$
r^{g} \cdot x_{t}=\sum_{h=1}^{H} n_{h, t-1} \cdot f_{h}^{L}\left(x_{t-1}, \ldots, x_{t-L}\right) \equiv \sum_{h=1}^{H} n_{h, t-1} \cdot f_{h, t}^{L}
$$

where $n_{h, t-1}$ denotes the fraction of investor type $h$ at the beginning of period $t$, before than the equilibrium price $x_{t}$ has been observed and $L$ is a random number of lags. Now the realized excess return over period $t$ to the period $t+1$ is computed by

$$
\mathbf{Z}_{t+1}=\mathbf{p}_{t+1}-r^{g} \cdot p_{t}
$$

We need now a measure of profits generated by forecasts. Let a performance measure $\pi_{h, t}$ be defined by

$$
\pi_{h, t}=E_{\iota}\left[\frac{\mathbf{Z}_{t+1} \cdot \rho_{h, t}}{a \cdot \sigma_{t}^{2}}\right]
$$

where

$$
\rho_{h, t}=E_{h, t}\left[\mathbf{Z}_{t+1}\right]=f_{h, t}^{\mathrm{L}}-r^{g} \cdot x_{t}=f_{h, t}^{\mathrm{L}}-\sum_{j=1}^{H} n_{j, t} \cdot f_{j, t}^{\mathrm{L}}=f_{h, t}^{\mathrm{L}} \cdot\left(1-\sum_{j \neq 1}^{H} n_{j, t} \cdot f_{j, t}^{\mathrm{L}}\right),
$$

So the $\pi$-performance is given by the realized performance for the $h$-investor. Let the updated fractions $n_{h, t}$ be given by the discrete choice probability (Gibb's distribution)

$$
n_{h, t}=\exp \left(\beta \cdot \pi_{h, t-1}\right) / Y_{t-1}
$$

where

$$
Y_{t}=\sum_{j=1}^{H} \exp \left(\beta \cdot \pi_{j, t}\right)
$$

The parameter $\beta$ is an intensity of choice. The parameter $\beta$ is a measure of investor's rationality. If the intensity of choice is infinite $(\beta=+\infty)$, the entire mass of investors uses the strategy that has the highest performance. If the intensity of 
choice is zero, the mass of investors distributes itself evenly across the set of available strategies. All forecasts will have the following form

$$
f_{t}^{\mathrm{L}}=g \cdot\left(x_{t-1}+\ldots+x_{t-1}\right)+b
$$

where the $g$ denotes the trend of investor forecasts and the $b$ denotes the bias of investor forecasts. If $b=0$, investor is called a pure trend chaser if $g>0$ and a contrarian if $g<0$. If $g=0$, investor is called purely biased. Investor is upward (downward) biased if $b>0$ $(b<0)$. In the special case $g=b=0$, investor is called fundamentalist, i.e., the investor believes that price return to their fundamental value. Fundamentalists strategy is based on all past prices and dividends in their information set, but they do not know the fractions $n_{h, l}$ of the other belief types.

\section{Simulations and the WOA}

For simulation of the financial market patterns, an updated version of the WOA is used. The algorithm replaces zero, one, two, three, four, five, six, and eight strategies with the lowest performances by sequel $0,1,2,3,4,5,6$, and 8 new strategies (i.e. OWOA, 1WOA, 2WOA, 3WOA, 4WOA, 5WOA, 6WOA, and 8WOA). A set of all strategies used for the simulation is composed from fifteen different strategies with specific parameters for probability distributions of stochastic processes. A replacement ratio of the market strategies is from $0 \%$ to $53.3 \%$. The high replacement ratio is implemented for a simulation the dramatic changes in the mood on the financial market are considered. From such conditions on the financial market, there is a bigger chance of the price turbulence emergence. The WOA replaces periodically the trading strategies that have the lowest performance level of strategies presented on the market by the new ones. The new strategies that enter the market are taken from the set that has the same stochastic parameters as the initial strategies, i.e., the trend has a normal distribution with the mean is equal 0 , and variance is equal $0.16(g \sim N(0,0.16))$, the bias has a normal distribution with the mean is equal 0 , and variance is equal 0.09 $(b \sim N(0,0.09))$, the memory length has an uniform distribution on a range of integers 1 , $2, \ldots, 100(m \sim U(1,100))$. Simulations are performed with fifteen agents represented by trading strategies. The intensity of choice, $\beta$, is equated to 120 . The WOA makes the replacement after 40 iterations. For example, when we want to replace four strategies with the lowest performance (4WOA, replacement ratio is $26.6 \%$ ) the algorithm after every 40 iterations evaluates and arranges in descending order the performance of all fifteen strategies in the market and the last four replaces by the new ones. Number of observations in our simulations is equal 8192. All simulations in our experiments were executed by Mathcad ${ }^{\circledR}$ software.

For a better understanding of the financial market dynamics with the WOA, we compare eight cases that differ in the replacement ratio. The first one is without WOA (0WOA, replacement ratio is $0 \%$ ), the last one replaces eight strategies (8WOA, replacement ratio is $53.3 \%$ ). 


\section{Price Returns Time Series Analysis}

For estimating and analysing the correlation structures on financial markets, a nonparametric approach is used. H. E. Hurst discovered very robust nonparametric methodology, which is called rescaled range, or $\mathrm{R} / \mathrm{S}$ analysis that is used for estimating the Hurst exponent. The R/S analysis was used for distinguishing random and non-random systems, the persistence of trends, and duration of cycles (Peters, 1994). This method is very convenient for an identification of fractal time series. Starting point for the Hurst's coefficient was the Brownian motion as a primary model for random walk processes. If a system of random variables is an independent identical distributed then $\mathrm{H}=0.5$. The values of Hurst exponent, belonging to $0<\mathrm{H}<0.5$, signify an anti-persistent system. Such a system must reverse itself more frequently than a random process can represent. This behaviour is very close to a mean-reverting process. The values of Hurst exponent belonging to $0.5<\mathrm{H}<1$, signify a persistent process that is characterized by long memory effects. This long memory occurs regardless of time scale, i.e., there is no characteristic time scale, which is the key characteristic of fractal time series (see Peters, 1994; Los, 2003; and Percival, Walden, 2000).

For the 0WOA case (no replacement of strategies), presented market strategies are generated randomly and the Hurst exponent, as we expect, is close to the Efficient Market Hypothesis (EMH) case, i.e., 0.5. When the WOA is implemented, we can see a strong learning effect that is transformed to long-memory (persistent) behaviour of price returns.

\section{Figure 1}

The Value of the Hurst Exponent with Different Replacement Rate of the WOA

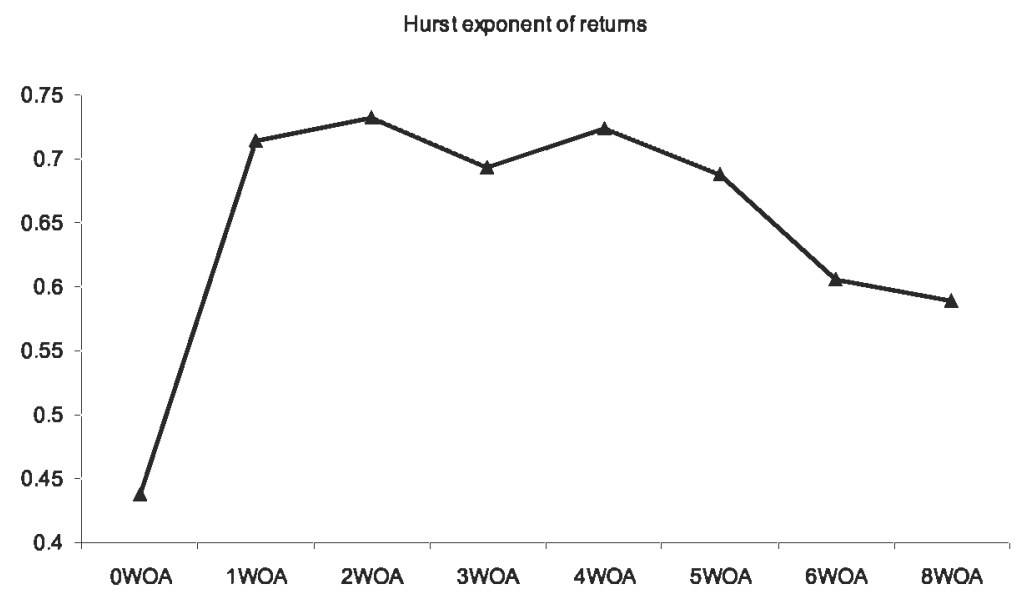

A highest level of persistence is in the 2 WOA case (13.3\% replacement rate) where the financial market has enough time to learn. If a number of replaced strategies is higher than before the learning effect is weakened by the randomly chosen new strategies that appear on the financial market. With higher replacement ratio, the value of the Hurst 
exponent declines as the learning is "diluted" by new strategies that enter randomly on the financial market. This phenomenon takes place from the $5 \mathrm{WOA}$ case to the $8 \mathrm{WOA}$ case (33\%- $53 \%$ the replacement rate; see Figure 1 and Table 1). Figure 2 shows the kurtosis of the price returns time series. A high kurtosis is, in the 1WOA case, followed by falling trend in the kurtosis value as the replacement ratio increases. The reason why we can observe a decreasing trend of the kurtosis value is the fall of the learning effect, which is caused by a higher proportion of new incoming strategies (randomly generated) on the financial market. Similar, but not so strong, results are in the R/S analysis in Figure 1. Variance of the price returns time series is depicted in Figure 3. We can observe rising trend as the replacement ratio increases. The higher is a number of incoming strategies the higher is a price volatility. Such a high fluctuation of strategies causes that the dynamic system representing the simulated financial market has not time to stabilize.

Table 1

Hurst Exponent, Kurtosis and Variance of the Simulated Time Series Price Returns

\begin{tabular}{|l|r|r|r|r|r|r|r|r|}
\hline & OWOA & 1WOA & 2WOA & 3WOA & 4WOA & 5WOA & 6WOA & 8WOA \\
\hline Hurst & 0.438 & 0.714 & 0.732 & 0.693 & 0.724 & 0.687 & 0.605 & 0.589 \\
\hline Kurtosis & -1.2 & 56.6 & 27.7 & 7.9 & 21.7 & 10.0 & 4.8 & 4.2 \\
\hline Variance & 0.016 & 0.017 & 0.024 & 0.034 & 0.025 & 0.036 & 0.066 & 0.064 \\
\hline
\end{tabular}

Figure 2

Value of Price Returns Kurtosis versus Different Replacement Rates of the WOA

Kurtosis of retums

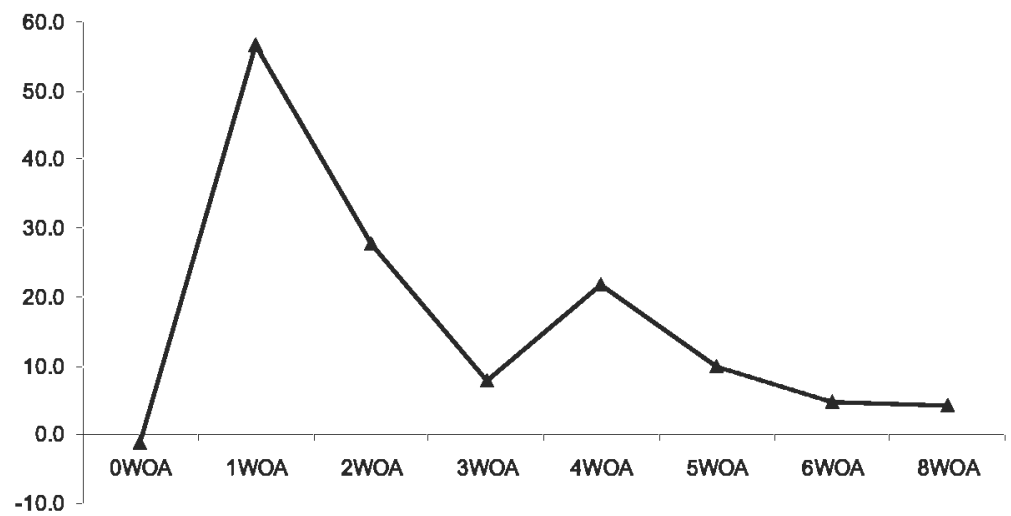

\section{A Wavelet Decomposition of the Time Series}

Wavelets are a relatively new tool for analysing time series. A wavelet analysis is in some case complementary to the existing analysing techniques, such as a correlation and spectral analysis. There are two main versions of the wavelet analysis. The first one is the continuous wavelet transform, CWT, which is designed to work with time series 
defined over the entire real axis. The second one is the discrete wavelet transform, DWT, which is suitable for time series defined over a range of integers, i.e., the DWT is a natural tool for discrete time series analysis. The wavelet transform decomposes 1-dimensional time series into 2-dimensional time-scale (frequency) space. In particular, while Fourier analysis breaks down a time series into constituent orthogonal sinusoids of different frequencies (constant periodicities), wavelet analysis breaks down such a time series into constituent orthogonal wavelets of different scales. A wavelet is a small wave that grows and decays essentially in a limited time period, in contrary a sine function is an example of a big wave oscillating on whole real axis (see Abramovich, Bailey, Sapatinas, 1999; and Los, 2003). A wavelet is a real-valued or complex function $\psi($.$) defined over the real axis (-\infty,+\infty)$ and satisfy admissibility condition. A wavelet $\psi($.) is said to be admissible if its Fourier transform

$$
\psi(f) \equiv \int \psi(u) e^{-i 2 \pi f u} d u
$$

is such that

$$
C_{\psi} \equiv \int_{0}^{\infty} \frac{|\psi(f)|^{2}}{f} d f<\infty,
$$

where $f$ is a frequency. Let $x($.$) be a function that satisfies \int_{-\infty}^{\infty} x^{2}(t) d t<\infty$. The condition (3.2) allows to reconstruct the function $x($.$) from its continuous wavelet transform (see$ Percival, Walden, 2000). There are other two basic properties that a wavelet satisfies. The first is that the integral of $\psi($.$) is zero:$

$$
\int_{-\infty}^{\infty} \psi(u) d u=0 .
$$

The second condition is that the square of $\psi($.$) integrates to unity:$

$$
\int_{-\infty}^{\infty} \psi^{2}(u) d u=1
$$

\section{Discrete Wavelet Transform}

The DWT is an important practical tool for financial time series analysis. The basic reasons are: an ability to re-express a time series in terms of coefficients that are associated with a particular time and a particular dyadic scale that we can also reconstruct a time series form its DWT coefficients. Given a real-valued time series $\left\{X_{t}: t=0,1, \ldots, N-1\right\}$, where the sample size $N$ is taken to be an integer multiple of, and let $j=1,2, \ldots, J_{0}$. Let us put $\mathbf{X}=\left(X_{0}, X_{1}, \ldots, X_{N-1}\right)$. The partial DWT of level $J_{0}$ of $\mathbf{X}$ is an orthonormal transform given by

$$
\mathbf{W}=W \mathbf{X},
$$


where $\mathbf{W}$ is an $N$ dimensional vector of the DWT coefficients, which includes both the discrete scaling coefficients, and the discrete wavelet coefficients. Here $\mathbf{W}$ is an $N \times N$ real-valued matrix associated with the orthonormal wavelet basis chosen, i.e., the matrix defining the DWT (if $N=2^{J}$ and $J_{0}=J$, we obtain a full DWT). Because of orthogonality of $\mathbf{W}$, the inverse discrete wavelet transform (IDWT) is given by:

$$
\mathbf{X}=W^{\prime} \mathbf{W}
$$

where $W^{\prime}$ denotes the transpose of $W$. The DWT coefficients $\mathbf{W}$ and matrix $W$ can partitioned such that

$$
\mathbf{W}=\left[\begin{array}{c}
\mathbf{W}_{1} \\
\mathbf{W}_{2} \\
\vdots \\
\mathbf{W}_{J_{0}} \\
\mathbf{V}_{J_{0}}
\end{array}\right] \quad W=\left[\begin{array}{c}
W_{1} \\
W_{2} \\
\vdots \\
W_{J_{0}} \\
V_{J_{0}}
\end{array}\right]
$$

i.e., $\mathbf{W}_{j}=W_{j} \mathbf{X}$ and $\mathbf{V}_{J_{0}}=V_{J_{0}} \mathbf{X} . \mathbf{W}_{j}$ is an $N_{j} \equiv N / 2^{j}$ dimensional vector of wavelet coefficients associated with changes at scale $\lambda_{j}=2^{j-1}, W_{j}$ is an $N_{j} \times N$ dimensional matrix, $\mathbf{V}_{\mathbf{J}_{\mathbf{0}}}$ is an $N^{J_{0}}$ dimensional vector of scaling coefficients associated with scale $\lambda_{J_{0}}, V_{J_{0}}$ is an $N_{J_{0}} \times N$ dimensional matrix. The time series $\left\{X_{t}: t=0,1, \ldots, N-1\right\}$ can be synthesized from $\mathbf{W}$ as

$$
\mathbf{X}=W^{\prime} \mathbf{W}=\sum_{j=1}^{J_{0}} W_{j}^{\prime} \mathbf{W}_{j}+V_{J_{0}}^{\prime} \mathbf{W}_{J_{0}} \equiv \sum_{j=1}^{J_{0}} D_{j}+S_{J_{0}},
$$

which defines the multiresolution analysis (MRA) of $x$ (.). Equation (3.8) is an additive decomposition in terms of the $N$ dimensional vectors $D_{j} \equiv W_{j}^{\prime} \mathbf{W}_{j}$ and $S_{J_{0}} \equiv V_{J_{0}} \mathbf{W}_{J_{0}}$ which are called the $j^{\text {th }}$ level detail and the $J_{0}^{\text {th }}$ level smooth that are associated with a particular scale $\lambda_{j}$ and $\lambda_{J_{0}}$ (see Percival, Walden, 2000). We can write the energy decomposition of $x($.$) as:$

$$
\|\mathbf{X}\|^{2}=\|\mathbf{W}\|^{2}=\sum_{j=1}^{J_{0}}\left\|\mathbf{W}_{j}\right\|^{2}+\left\|\mathbf{V}_{J_{0}}\right\|^{2},
$$

so that $\|\mathbf{W}\|^{2}$ represents the contribution to energy of the time series $x($.) due to changes at scale $\lambda_{j}$. The wavelet coefficients in the vector $\mathbf{W}_{j}$ are associated with differences in adjacent averages over a scale $\lambda_{j}=2^{j-1}$, while the scaling coefficient in $\mathbf{V}_{\boldsymbol{J}}$ is equal to $\sqrt{N}$ times the sample mean $\bar{x}$ of $X_{t}$ i.e., $\left\|\mathbf{V}_{J}\right\|^{2}=N \bar{x}^{2}$ (see Percival, Walden, 2000, page 62). Now we can decompose the sample variance of $x($.$) associated with particular scales$ $\lambda_{1}, \ldots \lambda_{J}$ as

$$
\hat{\sigma}_{x}^{2}=\frac{1}{N}\|\mathbf{X}\|^{2}-\bar{x}^{2}=\frac{1}{N}\|\mathbf{W}\|^{2}-\bar{x}=\frac{1}{N} \sum_{j=1}^{J}\left\|\mathbf{W}_{j}\right\|^{2}=\sum_{j=1}^{J} P_{W}\left(\lambda_{j}\right)
$$


where the sequence $P_{W}\left(\lambda_{j}\right)$ is the discrete wavelet spectrum. The orthonormality of the matrix $W$ implies that, for $1 \leq j, k \leq J$,

$$
D^{\prime}{ }_{j} D_{k}=\mathbf{W}_{j}^{\prime} W_{j} W_{k}^{\prime} \mathbf{W}_{k}=\left\{\begin{array}{l}
\mathbf{W}_{j}^{\prime} \mathbf{W}_{j} \quad k=j \\
0 \quad \text { otherwise. }
\end{array}\right.
$$

therefore $\left\|D_{j}\right\|^{2}=\left\|W_{j}\right\|^{2}$. We can rewrite the equation for the sample variance (3.10) on scale-by-scale basis as

$$
\hat{\sigma}_{x}^{2}=\frac{1}{N} \sum_{j=1}^{J}\left\|D_{j}\right\|^{2} \quad j=1, \ldots, J
$$

Figure 3

The Value of the Kurtosis of Price Returns Time Series with Different Replacement Rate of the WOA

Variance of returns

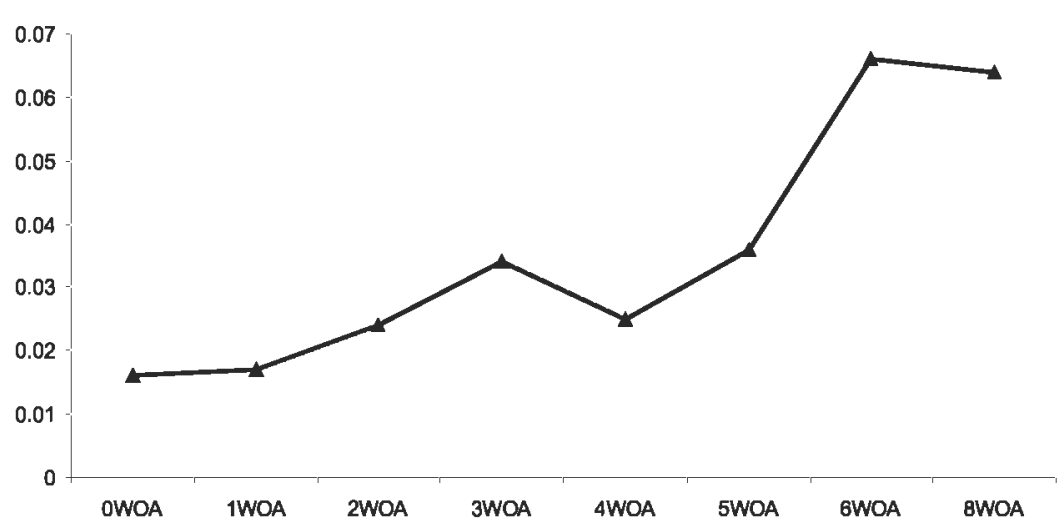

As an illustrative example we compare the $1 \mathrm{WOA}, 6 \mathrm{WOA}$ and $8 \mathrm{WOA}$ case. In Figure 4 we have a simulated price time series. The simulation were performed with the 1WOA i.e., low replacement ratio. At the left side of the Figure 4, around 2500 iteration there is a positive price jump. The price returns behaviour is analysed in a 6-scale wavelet detail vectors $\left(D_{1}, \ldots, D_{6}\right)$ decomposition showing that the dynamic phenomena are identifiable at all six scales, see Figure 5. This is in contrast to the 6WOA case (Figures 6,7) where the price turbulence (2000-3000 iterations) is mainly noticeable at the low scales. The 6 WOA case and the 8 WOA case, Figures 8, 9, have, in comparison to the lower replacement ratio cases, higher occurrence of significant price swings. This is also evident in Figures 4-9. 
Figure 4

Price Time Series, the 1WOA Case

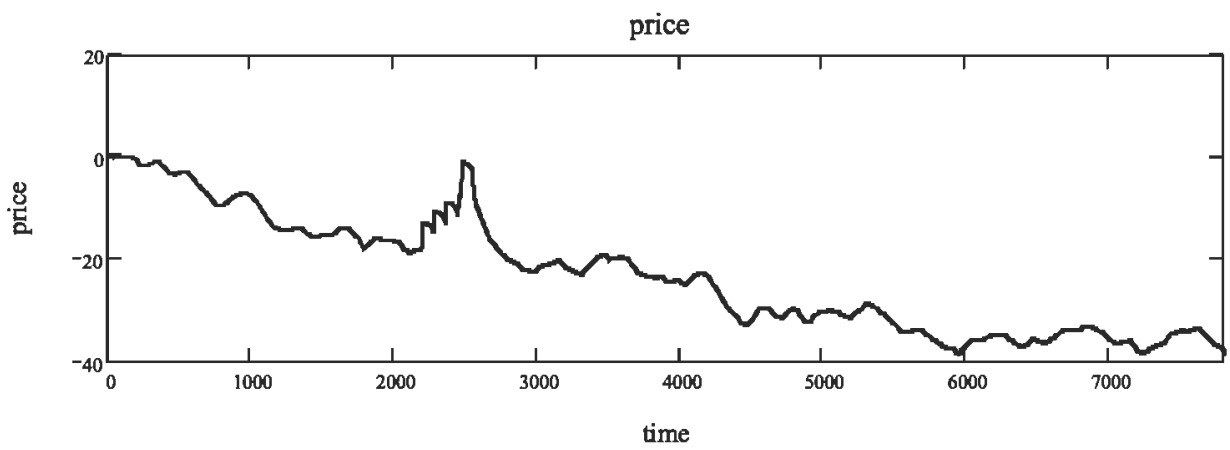

Figure 5

The 1WOA Case, a 6-Scale Wavelet Decomposition of the Returns Time Series, Where the High Frequency Wavelet Details Coefficients Are at the Top and the Low Frequency Wavelet Details Are at the Bottom

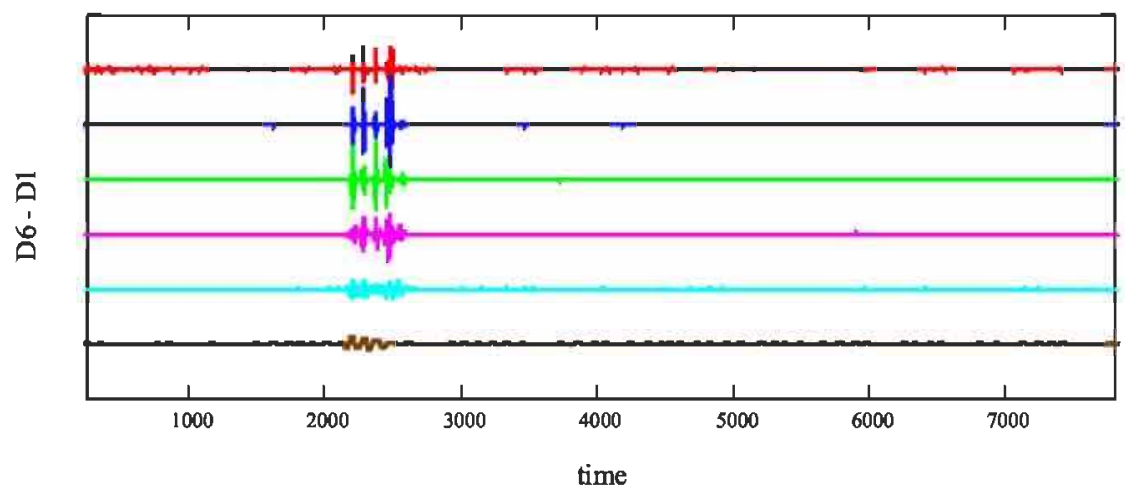

Figure 6

Price Time Series, the 6WOA Case

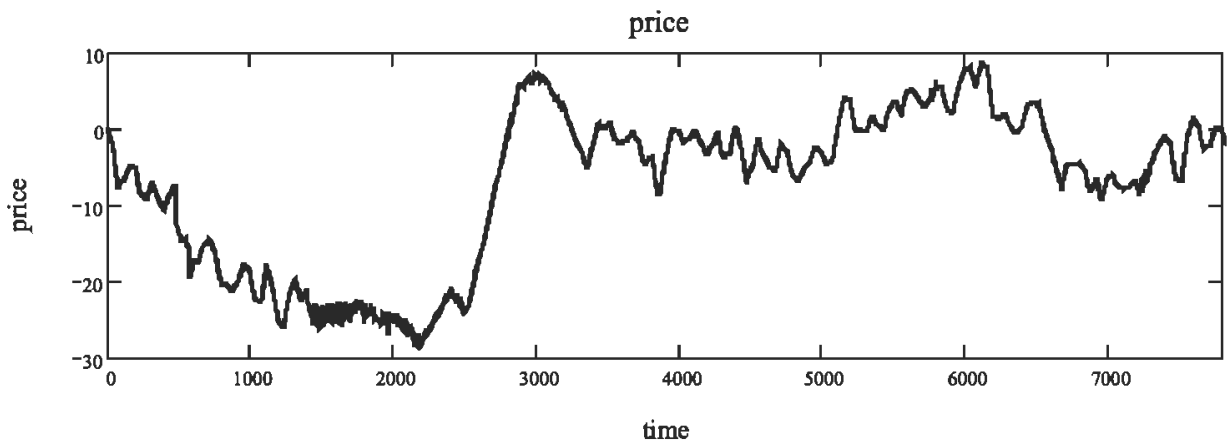


Figure 7

The 6WOA Case, a 6-Scale Wavelet Decomposition of the Returns Time Series, Where the High Frequency Wavelet Details Coefficients Are at the Top and the Low Frequency Wavelet Details Are at the Bottom

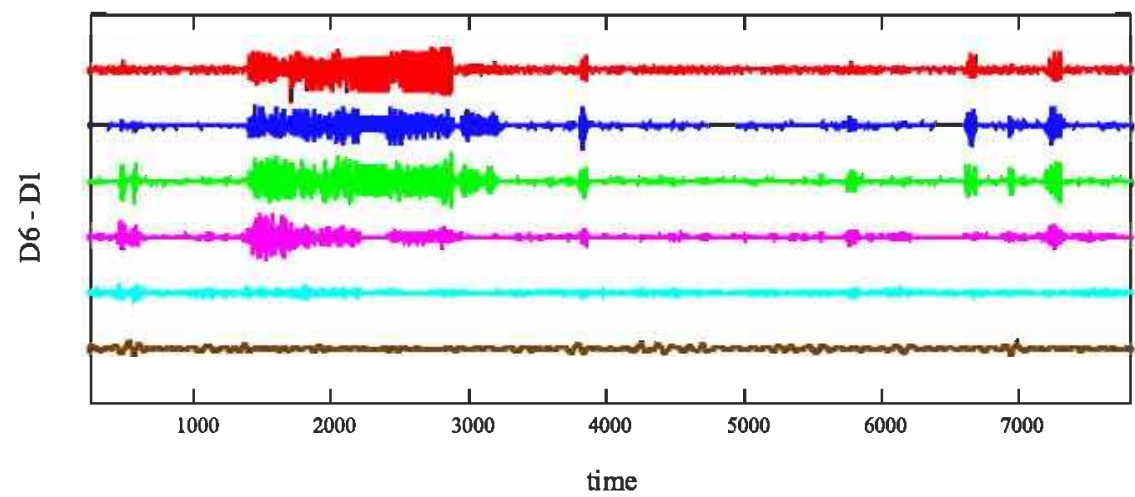

Figure 8

Price Time Series, the 8WOA Case

price

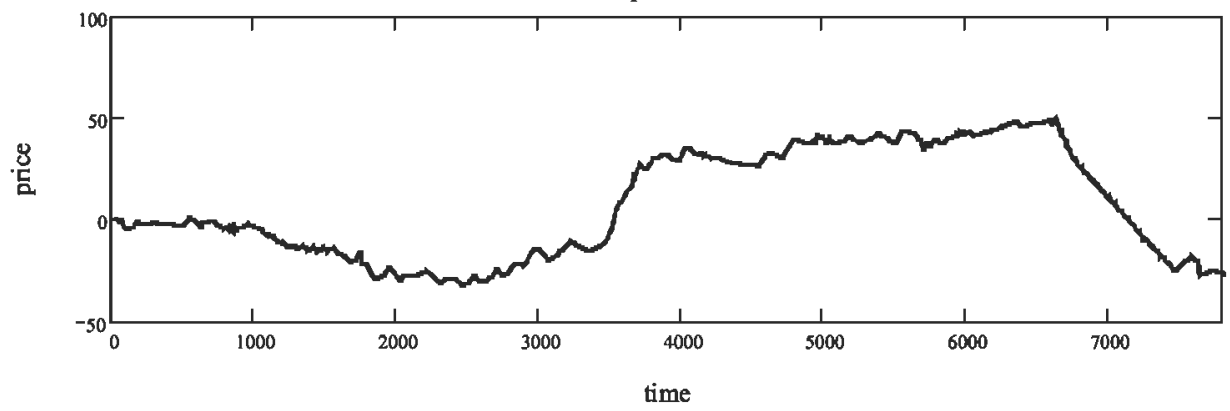




\section{Figure 9}

The 8WOA Case, a 6-Scale Wavelet Decomposition of the Returns Time Series, Where the High Frequency Wavelet Details Coefficients Are at the Top and the Low Frequency Wavelet Details Are at the Bottom

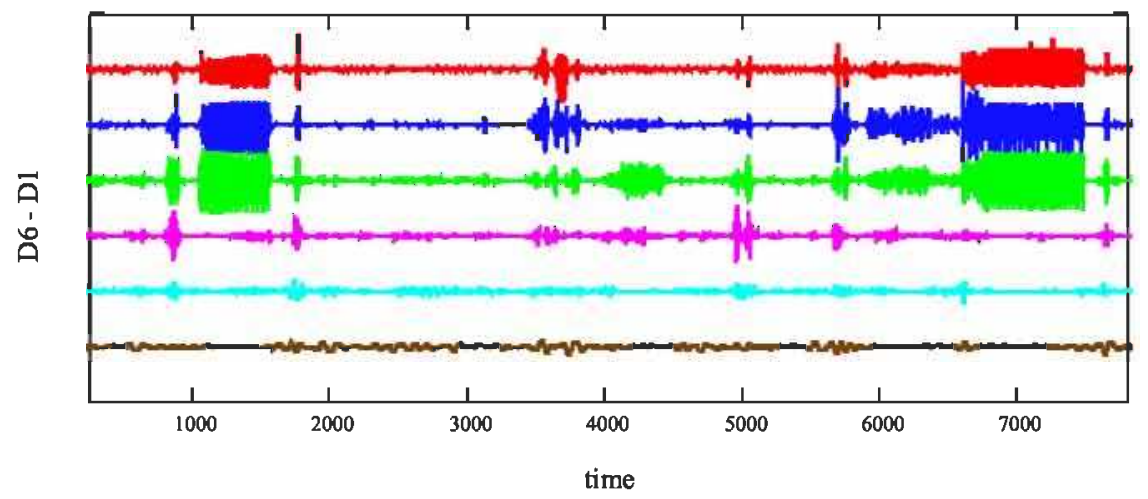

\section{Wavelet Variance Decomposition}

The wavelet variance decomposes a variance of the stochastic process on a scale basis and hence is important in financial time series processing. The wavelet variance is a succinct alternative to the power spectrum based on the Fourier transform. In this sense, the wavelet analysis can be seen as a refinement of the Fourier analysis. The key point is that the Fourier transform highlights a spectrum of a function or signal, but this frequency decomposition is global rather than localized. Conversely the wavelet transform offers localized a frequency decomposition. It provides information what frequency components are present and where they are occurring. Wavelets have significant advantages over basic Fourier analysis when the object under study is non-stationary and inhomogeneous (see Abramovich, Bailey, Sapatinas, 1999). There are alternatives to wavelets that provide localized frequency decomposition such as the windowed or short term Fourier transform, where the signal is restricted to an interval by multiplying it by a fixed window function. This process is repeated with shifted versions of the window function and the localized frequency information of the signal is obtained. In such case the window width is the same for all frequencies, so the amount of localization remains constant different frequencies. Wavelet analysis provides an alternative and preferable solution, especially in financial market time series, since it allows the degree of localization to be automatically and appropriately adapted. Large window widths are used for investigating low-frequency components, while small window widths are used for analysing high-frequency components. The wavelet variance decomposition yields a scale-based analysis that is often easier to interpret than the frequency-based spectrum (see Percival, Walden, 2000). Such decomposition helps us to track an evolution of the energy contribution at various scales, which is related to traders' investment horizons.

The following Figures 10 and 11 depict a scale energy decomposition, i.e., the decomposition of wavelet variance. For this decomposition we use a moving variance (window length 255) of the DWT multiresolution analysis detail vectors $D_{1}, \ldots, D_{6}$, see 
equations $3.8-3.12$. We are specifically interested in how the energy is changing on scales during the simulation, the 1WOA and 8WOA cases are examined. In Figure 10, the $1 \mathrm{WOA}$ case, we can see a dramatic change of market structure during simulations, there is a high increase of the energy at scales $D_{1}$ and $D_{2}$ in time 6000 . This increment lasts for about 200 iterations. In Figure 11, the 8WOA case, we can observe no such changes as in the 1 WOA case. In comparison to the 1 WOA case, the overall energy level at scales $D_{1}, D_{2}, D_{3}$ is higher (higher variance). This is caused mainly by higher replacement ratio of the WOA.

Figure 10

The 1WOA Case. Moving Sum of Squared Wavelet Detail Vectors (window length 255) of the Daublet (12) DWT Multiresolution Analysis

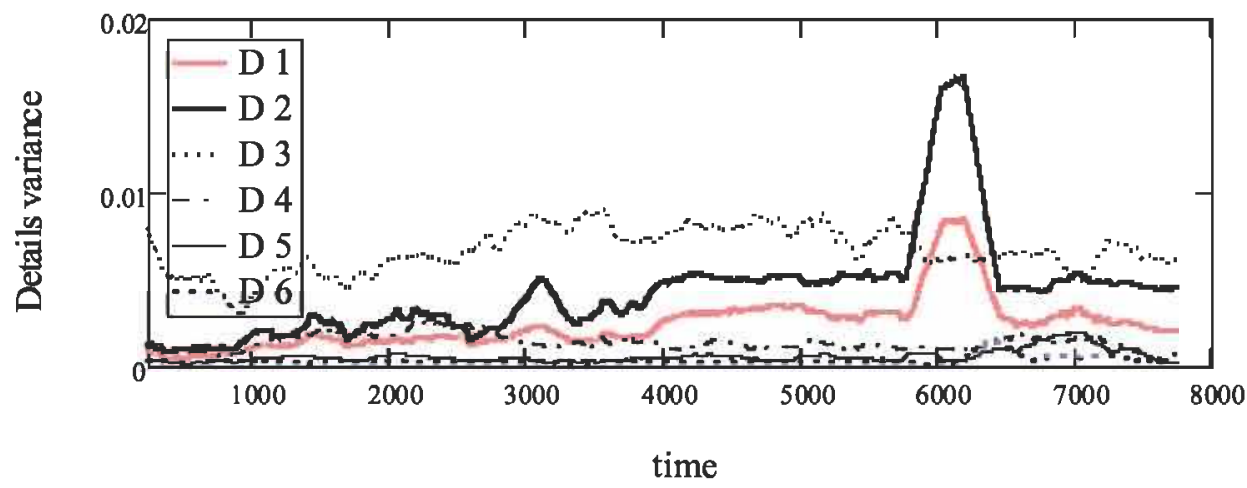

Figure 11

The 8WOA Case. Moving Sum of Squared Wavelet Detail Vectors (window length 255) of the Daublet (12) DWT Multiresolution Analysis

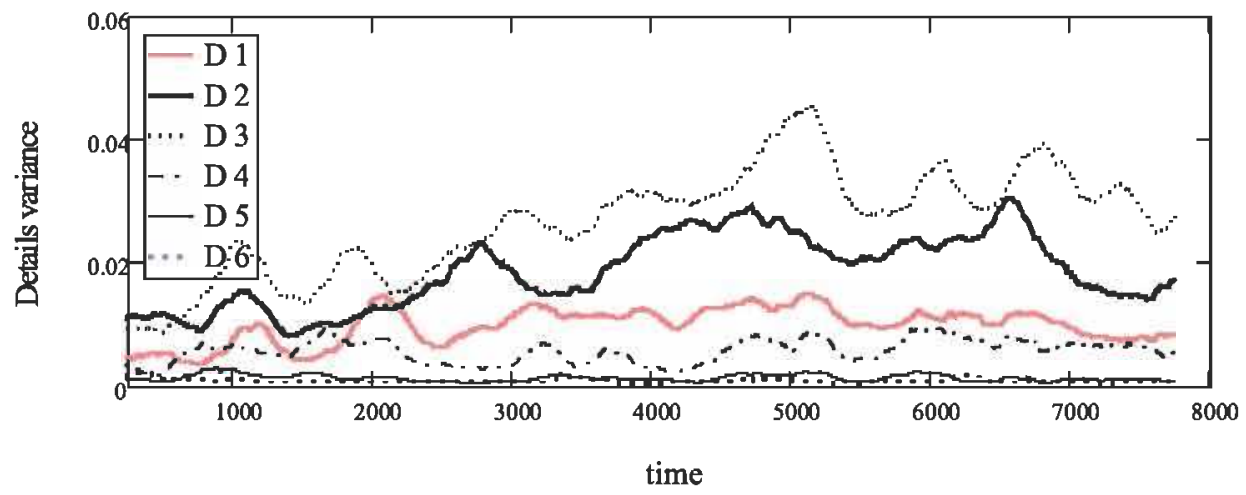




\section{Conclusions}

We demonstrate that the HAM considerably changes its behaviour when we implement the WOA. Due to an implementation of the WOA, we observe a considerable increase of the time series persistence, but when a number of the replaced strategies is increasing beyond some point, then the value of the Hurst exponent declines as the learning is "diluted" by new strategies that enter randomly on the financial market. We can also observe higher returns time series volatility as the replacement ratio rises.

An application of the wavelet variance is a very convenient tool for activity (energy) decomposition on scale basis. Our simulations show, that the higher replacement ratio (it causes an increment in the wavelet variance) the higher activity levels at all scales. A financial market with WOA (see Figures 10,11) allocates most the market energy in the high frequencies (low scales). In this situation newly coming strategies bring into innovations. These innovations cause an expansion of the price time series volatility. The R/S analysis and wavelet transforms enable us to reformulate the efficient market hypothesis by a behaviour of the Hurst exponent, the kurtosis of the returns, the variance of the returns, and the variance of wavelet resonance coefficients of the returns time series.

\section{References}

Abramovich F., Bailey T. C., Sapatinas T. (1999), "Wavelet Analysis and its Statistical Applications." J. R. Statist. Soc. D, 48.

Brock, W. A., Hommes, C. H. (1997), "A Rational Route to Randomness." Econometrica.

Brock, W. A., Hommes, C. H. (1998), "Heterogeneous Beliefs and Routes to Chaos in a Simple Asset Pricing Model." Journal of Economic Dynamics and Control, 22.

Brock, W. A. (2001), "Growth Theory, Nonlinear Dynamics and Economic Modeling." Edward Elgar.

Chiarella, C. (1992), "The Dynamics of Speculative Behaviour." Annals of Operational Research, 37.

Chiarella, C., He, X. (2000), "Heterogeneous Beliefs, Risk and Learning in a Simple Asset Pricing Model with a Market Maker. Quantitative Finance Research Group." University of Technology Sydney, Research Paper No. 35.

Gaunersdorfer, A. (2000), "Endogenous Fluctuations in a Simple Asset Pricing Model with Heterogeneous Agents." Joumal of Economic Dynamics and Control, 24.

Gençay, R., Selçuk, F., Whitcher, B. (2002), "An Introduction to Wavelets and Other Filtering Methods in Finance and Economics." Academic Press.

Haltiwanger, J., Waldman, M. (1985), "Rational Expectations and the Limits of Rationality: an Analysis of Heterogeneity." American Economic Review, 75.

Hommes, C. H. (2006), "Heterogeneous Agent Models in Economics and Finance," in Tesfatsion, L., Judd, K. L. (eds.), Handbook of Computational Economics, Volume 2: Agent-Based Computational Economics. Elsevier Science B.V.

Los, C. A. (2003), "Financial Market Risk: Measurement and Analysis." Routledge International Studies in Money and Banking. London, UK : Taylor and Francis Books Ltd.

Lucas, R. E. (1978), "Asset Prices in an Exchange Economy." Econometrica, 46.

Lorenz, H.-W. (1993), "Nonlinear Dynamical Economics and Chaotic Motion." Berlin : Springer Verlag.

Lux, T. (1997), "Economics Dynamics. Phase Diagrams and their Economic Application." Cambridge : Cambridge University Press.

Mallat, S. (1998), “A Wavelet Tour of Signal Processing." Academic Press.

Percival, D. B., Walden, A. T. (2000), "Wavelet Methods for Time series Analysis." Cambridge : Cambridge University Press.

Peters, E. (1994), "Fractal Market Analysis, Applying Chaos Theory to Investment and Economics." Springer. 
Vácha, L., Vošvrda, M. (2005), "Dynamical Agents' Strategies and the Fractal Market Hypothesis." Prague Economic Papers, 14(2), pp. 172-179. ISSN 1210-0455.

Vošvrda, M. (2001), "Bifurcation Routes in Financial Markets." 19th International Conference Mathematical Methods in Economics 2001, Hradec Králové.

Vošvrda, M., Vácha L. (2002 a), "Heterogeneous Agent Model with Memory and Asset Price Behaviour." Proceedings of the 20th International Conference Mathematical Methods in Economics 2002, Ostrava, pp. 273-282.

Vošvrda, M., Vácha L. (2002 b), "Heterogeneous Agent Model and Numerical Analysis of Learning." Bulletin of the Czech Econometric Society, No. 17, pp. 15-22. ISSN 1212-074X.

Vošvrda, M., Vácha L. (2003), "Heterogeneous Agent Model with Memory and Asset Price Behaviour." Prague Economic Papers, (12)2, pp. 155-168, ISSN 1210-0455.

\section{APPENDIX \\ Discrete Wavelet Filters}

The discrete wavelet and scaling filters which we use in the pyramid algorithm. We begin with the wavelet filter, that is a high-pass filter; let $\left\{h_{l}: l=0, \ldots, L-1\right\}$ be a real-valued wavelet filter, where $L$ is the width of the filter and must be an even integer. For $\left\{h_{l}\right\}$ with the width $L$, we must have $h_{0} \neq 0$ and $h_{L-1} \neq 0$, then we define $h_{l}=0$ for $l<0$ and $l \geq L$, in fact $\left\{h_{l}\right\}$ is an infinite sequence with at most $L$ nonzero values. A wavelet filter must satisfy the following three basic properties, (Percival, Walden, 2000):

$$
\sum_{l=0}^{L-1} h_{l}=0, \quad \sum_{l=0}^{L-1} h_{l}^{2}=1 .
$$

and

$$
\sum_{l=0}^{L} h_{l} h_{l+2 n}=\sum_{l=-\infty}^{\infty} h_{l} h_{l+2 n}=0 \quad n=1,2, \ldots
$$

i.e., a wavelet filter must sum up to zero, must have unit energy and must be orthogonal to its even shifts. We call the last two properties as the orthonormality properties of a wavelet filters. The second filter is the low-pass filter. Coefficients of the filter are determined by the quadrature mirror relationship, the filter is called the quadrature mirror filter $(\mathrm{QMF})^{1}\left\{g_{l}\right\}$ that corresponds to $\left\{h_{l}\right\}$ :

$$
g_{l}=(-1)^{l+1} h_{L-1-l} \quad l=0, \ldots, L-1 .
$$

The filter $\left\{g_{l}\right\}$ is known as a scaling filter. The wavelet and scaling filters both satisfy the orthonormality property, i.e., the unit energy and orthogonality to its even shifts.

Coiflet and Daublet are orthogonal discrete wavelets designed by Ingrid Daubechies. Translations and dilatations of the Coiflet wavelet filter are depicted in Figure A.1.

1 The sum of magnitude response of the high-pass and the low-pass filters is equal to one at every frequency. The QMFs have an ability for the perfect reconstruction of a signal without aliasing effects. 


\section{Figure A1}

Translation (upper row) and Dilatation (lower row) of the Coiflet (12) Wavelet

Coiflet (12) wavelet

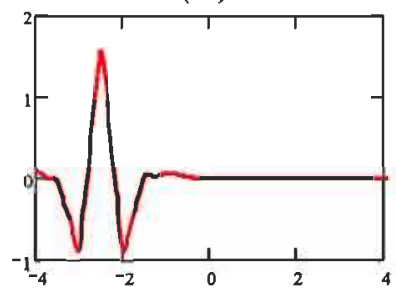

Coiflet (12) wavelet

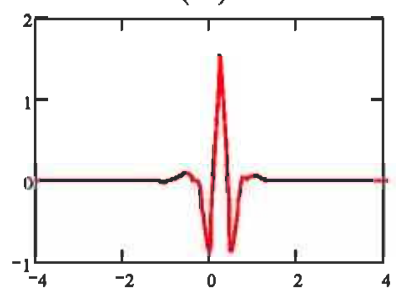

Coiflet (12) wavelet

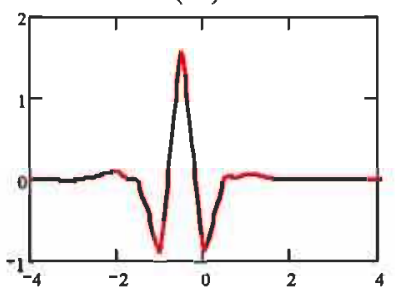

Coiflet (12) wavelet

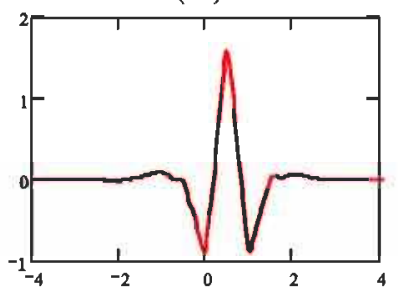

Coiflet (12) wavelet

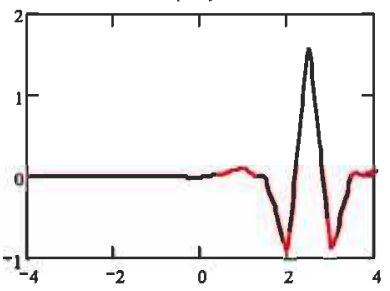

Coiflet (12) wavelet

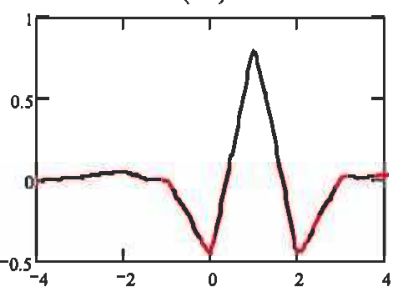

\section{Pyramid Algorithm}

In practice, we do not compute the product of the $N \times N$ dimensional matrix $W$ as the vector $\mathbf{X}$ which involves $N^{2}$ multiplications, but we use the pyramid algorithm developed by Mallat (1998). The DWT pyramid algorithm is even faster than the fast Fourier transform algorithm. The algorithm filters the input data vector $\mathbf{X}$ using the wavelet filter $\left\{h_{l}\right\}$ and the scaling filter $\left\{g_{l}\right\}$, subsamples both filter outputs to half their original lengths, keeps the subsampled output from the wavelet filter as wavelet coefficients and repeats the above filtering operations on the subsampled output from the scaling filter. 